\title{
Kinetic Study on the Heavy Metal lons Removal from Aqueous Solutions using multi-walled carbon nanotubes
}

\author{
Junjie Chen* and Baofang Liu
}

School of Mechanical and Power Engineering, Henan Polytechnic University, Jiaozuo, Henan, China

comcjj@yeah.net, baofang@escience.cn

\begin{abstract}
Keywords: Adsorption; Heavy metal ions; Aqueous solutions; Kinetic model; Multi-walled carbon nanotubes.
\end{abstract}

\begin{abstract}
Kinetic study on the removal of zinc(II), copper(II), lead(II) and cadmium(II) from the aqueous solutions using multi-walled carbon nanotubes (MWNTs) was carried out to examine the temperature effect on the adsorption of zinc(II), copper(II), lead(II) and cadmium(II) as well as to explore the potentiality of using carbon nanotubes as a promising adsorbent for environmental remediation. Multi-walled carbon nanotubes were characterized by BET (Brunauer-Emmett-Teller), FE-SEM (Field emission scanning electron microscopy), and DPASV (differential pulse anodic stripping voltammetry). Adsorption experiments were carried out and comparisons with the previous work were made. Experimental results showed that the multi-walled carbon nanotubes can successfully remove zinc(II), copper(II), lead(II) and cadmium(II) from aqueous solutions. Increasing solution temperature can significantly improve the removal efficiency because of the endothermic nature of adsorption process. The kinetics of zinc(II), copper(II), lead(II) and cadmium(II) adsorption on multi-walled carbon nanotubes were analyzed, and the calculation results showed that the heavy metal ions adsorption is a pseudo-second-order process, and its capacity increases with increasing solution temperature. The binding of the metal ions by the multi-walled carbon nanotubes was evaluated from the adsorption capacities and found in the following order: copper(II) $>$ lead(II) $>$ zinc(II) $>$ cadmium(II). Finally, multi-walled carbon nanotubes demonstrated that they are a promising adsorbent for the removal of heavy metal ions from aqueous solutions.
\end{abstract}

\section{Introduction}

Heavy metal pollution sources include environmental, hobbies, industrial, water supply, and others. $\mathrm{Zn}, \mathrm{Cu}, \mathrm{Pb}, \mathrm{Cd}, \mathrm{Cr}, \mathrm{Hg}$, and $\mathrm{As}$ are the most common toxic heavy metals in water system; therefore, the pretreatment of wastewater containing heavy metal will be required [1]. To improve water quality for effluents from industrial facilities and wastewater treatment, increasing awareness for development of systems has provided incentives to improve performance of existing technologies, or develop new technologies. Various methods exist for the removal of metal ions from aqueous solutions, but adsorption is by far the widely used and most versatile process [2].

Carbon nanotubes (CNTs) are relatively new materials with a constantly growing variety of applications because of their unique chemical, optical, mechanical and electrical properties. The above excellent properties make carbon nanotubes promising materials for a wide variety of applications. Examples include chemical sensors, catalyst supports, and hydrogen storage [3]. Since it has been demonstrated that carbon nanotubes possess potential for the removal of many kinds of pollutants from aqueous solutions due to their large surface areas along with their ability to establish $\pi-\pi$ electrostatic interactions, carbon nanotubes have been focused on widely in analytical chemistry and environmental protection in particularly [4].

Although carbon nanotubes exhibit great potential for the adsorption of heavy metal ions from aqueous solutions, the sensitivity, selectivity, and removal efficiency in particularly remain limited [5]. Therefore, the modification of carbon nanotubes is considered to be an important route for the sensitivity, selectivity, and removal efficiency enhancement of heavy metals [6]. Multi-walled carbon nanotubes consist of multiple rolled layers (concentric tubes) of graphene. The surfaces of 
multi-walled carbon nanotubes can be modified in a wide variety of applications. Examples include physical adsorption of the modifier to multi-walled carbon nanotubes surface or chemical bond formation between the modifying species and multi-walled carbon nanotubes surfaces [7]. Recent studies have focused on the removal of heavy metal ions using modified carbon nanotubes, including uranium, strontium and nickel, copper, chromium, lead, cadmium, as well as zinc, nickel, cadmium, and copper ions from aqueous solutions [8].

Although there are many investigations that focused on heavy metals removed from polluted water using carbon nanotubes [9], but exploring their adsorption on different types of carbon nanotubes from the kinetic point of view to reveal the possible adsorption mechanism for efficient removal is still lacking. Kinetic study is important to an adsorption process because it depicts the uptake rate of adsorbate, and controls the residual time of the whole adsorption process. Adsorption kinetics is the study of the adsorption process rate to understand the factors that affect the adsorption process. Kinetic study includes careful monitoring of the experimental conditions that affect the reaction rate and consequently help to attain equilibrium within a reasonable time range. Such studies yield information about the possible adsorption mechanism as well as the different transition states on the way to the formation of the final adsorbate-adsorbent complex and help to develop appropriate kinetic models to describe the adsorbate-adsorbent interactions. Once the reaction rates and the dependent factors are unambiguously known, the same can be utilized to develop adsorbent materials for industrial application and will be useful in understanding the complex dynamics of adsorption process. Currently, there are quite a few kinetic models used for adsorption description. These kinetic models could be classified as adsorption reaction models; which consider the adsorption as a global reaction, and adsorption diffusion models that adsorption occurs in three main consecutive steps; liquid film diffusion, followed by intra-particle diffusion and finally adsorption and desorption between the adsorbate and adsorbent active sites [10].

The main objective of present work is to kinetically study the adsorption of zinc(II), copper(II), lead(II) and cadmium(II) from aqueous solutions using multi-walled carbon nanotubes and consequently to explore the potentiality of using carbon nanotubes as a promising adsorbent for environmental remediation. Particular objectives are to examine the temperature effect on the adsorption of zinc(II), copper(II), lead(II) and cadmium(II) using multi-walled carbon nanotubes. Comparisons with the previous work of Kosa et al. [11] were made. Kosa et al. [11] modified multi-walled carbon nanotubes with 8-hydroxyquinoline to use for the removal of heavy metals from aqueous solutions, to study the Recycling and regeneration of the used MWCNTs, and to optimize the desorption conditions. However, in the present work, different multi-walled carbon nanotubes and characterization method were employed; in addition, different kinetic mathematical models and mechanisms were used and evaluated.

\section{Experimental}

\section{Materials}

Multi-walled carbon nanotubes were used as received, with average diameter between 20 and 40 nm. Analytical-grade zinc, copper, lead and cadmium nitrate were employed to prepare stock solutions, containing $100 \mathrm{mg} / \mathrm{L}$ of zinc(II), copper(II), lead(II) and cadmium(II), which were further diluted with deionized (DI) water to the required metal ion concentration for the adsorption measurements.

\section{Characterization techniques}

The pore diameter, pore specific volume and BET specific surface area were measured by nitrogen adsorption/desorption at $77.4 \mathrm{~K}$ using a Micrometrics ASAP 2020MP instrument. FE-SEM was performed on a SUPRA ${ }^{\mathrm{TM}} 55 \mathrm{VP}$ electron microscope.

\section{Analytical measurements}

The total metal ion concentration was determined by voltammetric measurement using a Metrohm, 797 VA computrace with a three electrode system, including hanging mercury as the working 
electrode, a platinum plate as the counter electrode, and $\mathrm{Ag} / \mathrm{AgCl}(3.0 \mathrm{~mol} / \mathrm{L} \mathrm{KCl})$ as the reference electrode. The metal ion determination was evaluated by DPASV.

\section{Adsorption experiments}

Kinetic experiments were conducted to establish the temperature effect on the adsorption process and consequently to identify the adsorption rate.

All batch adsorption experiments were carried out using $250 \mathrm{~mL}$ polyethylene bottles with addition of $125 \mathrm{mg}$ of multi-walled carbon nanotubes and $100 \mathrm{ml}$ aqueous solutions containing $0.5 \mathrm{mg} / \mathrm{L}$ of zinc(II), copper(II), lead(II) and cadmium(II) with the appropriate $\mathrm{pH}$. The polyethylene bottles were placed in an ultrasonic bath, which was operated at defined temperatures and times. The temperature in an ultrasonic bath was maintained using a recirculating water system. The aqueous samples were filtered through a $0.2 \mu \mathrm{m}$ PTFE (polytetrafluoroethylene) membrane filter and the concentrations of metal ions in filtrate were analyzed using differential pulse anodic stripping voltammetry. Note that the adsorption of metals ions on the walls of the glass flasks and the membrane filter was determined by running the blank experiments without multi-walled carbon nanotubes and could be ignored.

\section{Results and discussion}

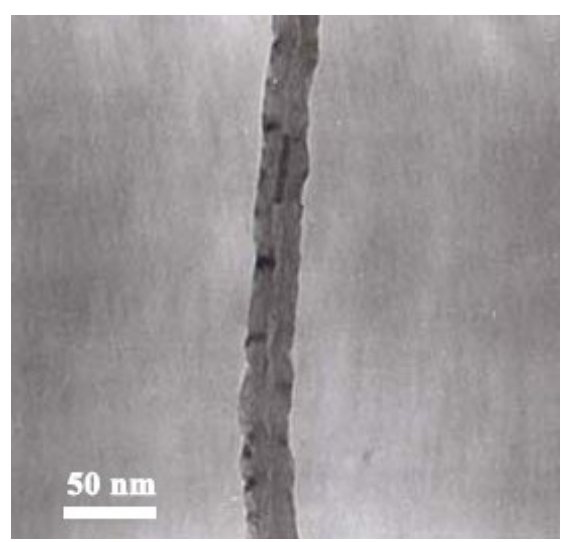

Fig. 1. Transmittance electron microscope image for the multi-walled carbon nanotubes.

Fig. 1 shows the representative transmittance electron microscope (TEM) image for the multi-walled carbon nanotubes. TEM image reveals the morphological structure of the multi-walled carbon nanotubes. The average diameter of multi-walled carbon nanotubes is between 20 and $40 \mathrm{~nm}$. The TEM analysis verifies the hollow structure of multi-walled carbon nanotubes used and reveals that the inner diameter is between 2 and $8 \mathrm{~nm}$.

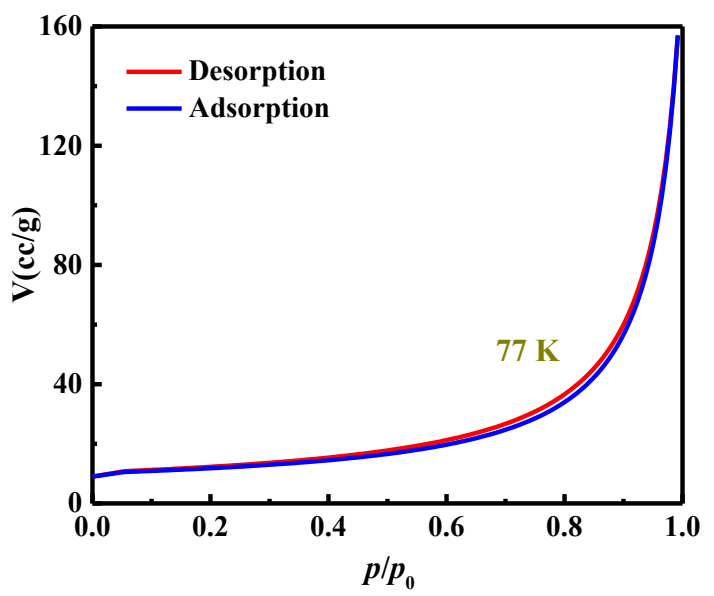

Fig. 2. Nitrogen adsorption/desorption isotherms of multi-walled carbon nanotubes.

The nitrogen adsorption/desorption isotherms are determined from a nitrogen adsorption isotherm measured at $77 \mathrm{~K}$. Fig. 2 shows the nitrogen adsorption/desorption isotherms of multi-walled carbon 
nanotubes. The specific surface area is $58.0 \mathrm{~m}^{2} / \mathrm{g}$, with a calculation using the Brunauer-Emmett-Teller equation-specific surface areas.

Figs. 3 and 4 show the results of kinetics study, i.e., the temperature effect on the removal efficiency of zinc(II), copper(II), lead(II) and cadmium(II) from aqueous solutions using multi-walled carbon nanotubes. The results of kinetics study demonstrate that increasing solution temperature enhances the adsorption process, indicating the endothermic nature of adsorption process. In terms of copper(II), increasing solution temperature has little effect on removal efficiency, close to perfect removal. In the case of zinc(II), lead(II) and cadmium(II), the adsorption reaches equilibrium after 20 min, irrespective of solution temperature, whereas in the case of copper(II), the adsorption reached equilibrium after 2 min at all temperatures, indicating the strong binding of copper(II) to multi-walled carbon nanotubes compared to the other metal ions. At $298 \mathrm{~K}$, the binding of the metal ions by multi-walled carbon nanotubes is as follow: copper(II) $>$ lead(II) $>$ zinc(II) $>$ cadmium(II). The high removal efficiency of copper(II) has been addressed earlier in previous work of Kosa et al. [11]: copper(II) usually form inner-sphere complexation, the copper(II) complexes stabilized by the Jahn-Teller effect, and its ability to be reduced by the carbonaceous surface. The lower adsorption of cadmium(II) might be because of its lower tendency to form hydrolysis products as well as because its ions do not compete effectively for variable charge surfaces, such as multi-walled carbon nanotubes, restricting its adsorption to permanently charged sites.
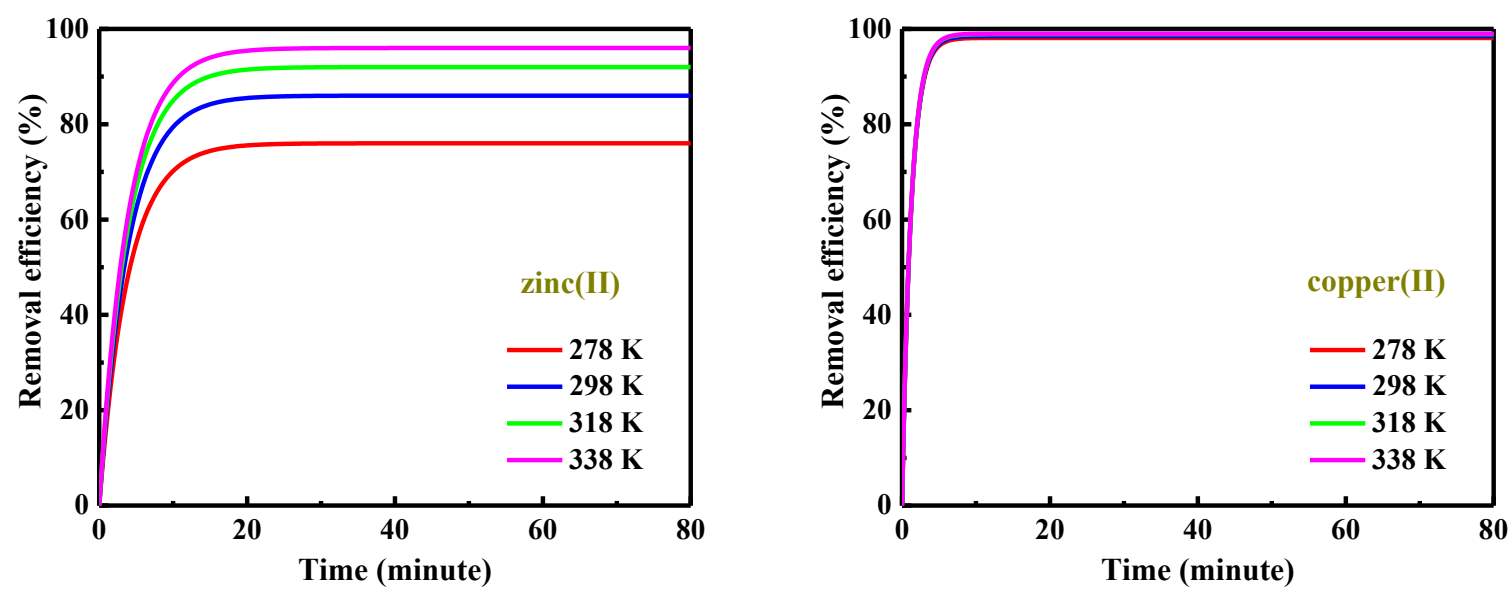

Fig. 3. The effect of solution temperature on the removal efficiency of zinc(II) and copper(II).
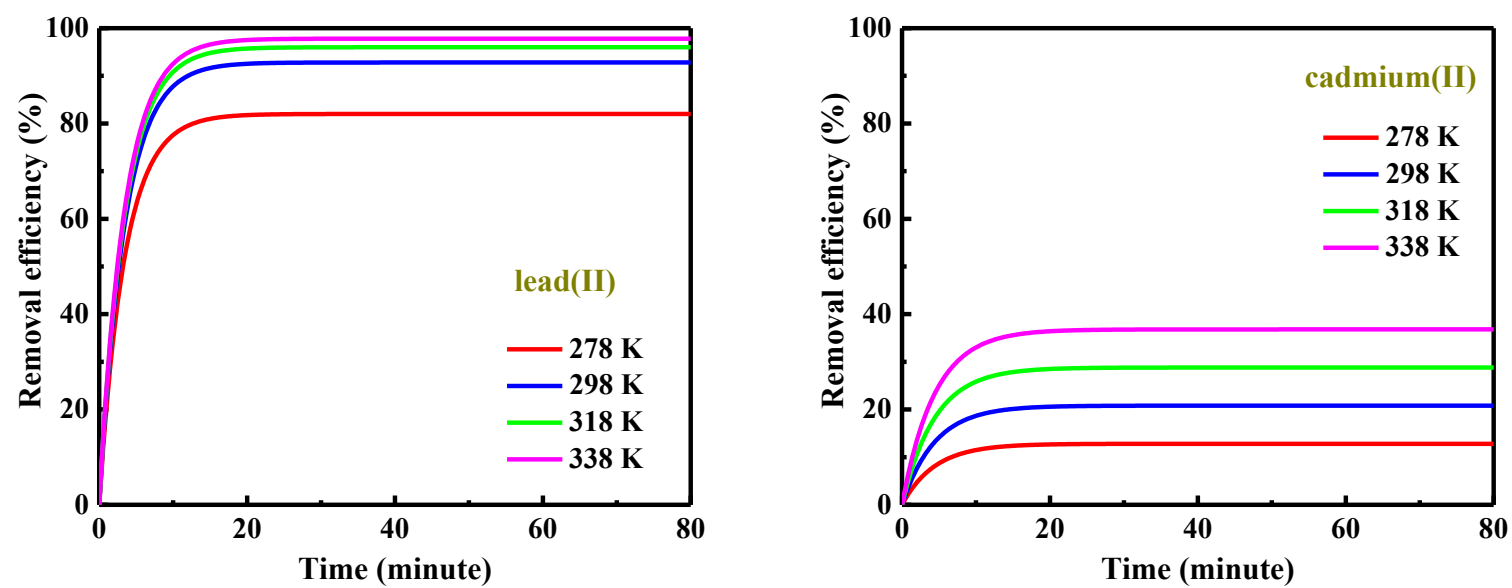

Fig. 4. The effect of solution temperature on the removal efficiency of lead(II) and cadmium(II).

Although the removal efficiency has been widely studied (such as the previous work of Kosa et al. [11]), the kinetic mathematical models and mechanisms are still lacking. Many kinetic models could be used to describe the adsorption of heavy metal ions by multi-walled carbon nanotubes from aqueous solutions at different temperatures. The kinetics of zinc(II), copper(II), lead(II) and 
cadmium(II) adsorption on multi-walled carbon nanotubes are analyzed using the kinetic models such as Elovich models [12], pseudo-first-order, pseudo-second-order [13], and fraction power function [14].

The pseudo-second-order equation is used to describe the adsorption of the zinc(II), copper(II), lead(II) and cadmium(II) on multi-walled carbon nanotubes. The pseudo-second-order model assumes that the adsorption might be second order [15], the rate limiting step may be chemical adsorption involving sharing or exchange of electrons between the solid adsorbent and divalent metal ions [16], and finally the adsorption follows the Langmuir equation [17].

The linearized form of the pseudo-second-order rate equation is as follow: $t / q_{t}=1 / k_{2} q_{e}{ }^{2}+t / q_{e}$. Where $k_{2}(\mathrm{~g} / \mathrm{mg} \cdot \mathrm{min})$ is the pseudo-second-order rate coefficient, and $q_{t}$ and $q_{e}$ are the values of the amount adsorbed per unit mass at any time $t$ and at equilibrium, respectively. The plot of $t / q_{t}$ and $t$ must give a linear relationship from which $k_{2}$ and $q_{e}$ can be estimated from the intercept and slope, respectively; calculation results show in Figs. 5 and 6. Applying the pseudo-second-order rate equation to the adsorption of zinc(II), copper(II), lead(II) and cadmium(II) experimental data converged very well for all the metal ions with excellent regression coefficients and straight lines. The above findings demonstrate the suitability of the pseudo-second-order rate equation for the description of the zinc(II), copper(II), lead(II) and cadmium(II) adsorption by multi-walled carbon nanotubes from aqueous solutions. In general, increasing solution temperature increases the amount adsorbed at equilibrium, indicating the endothermic nature of the adsorption process of the target heavy metal ions on multi-walled carbon nanotubes from aqueous solutions, which is consistent with other adsorbents such as silica-supported dithiocarbamate and multi-walled carbon nanotubes/chitosan composite.
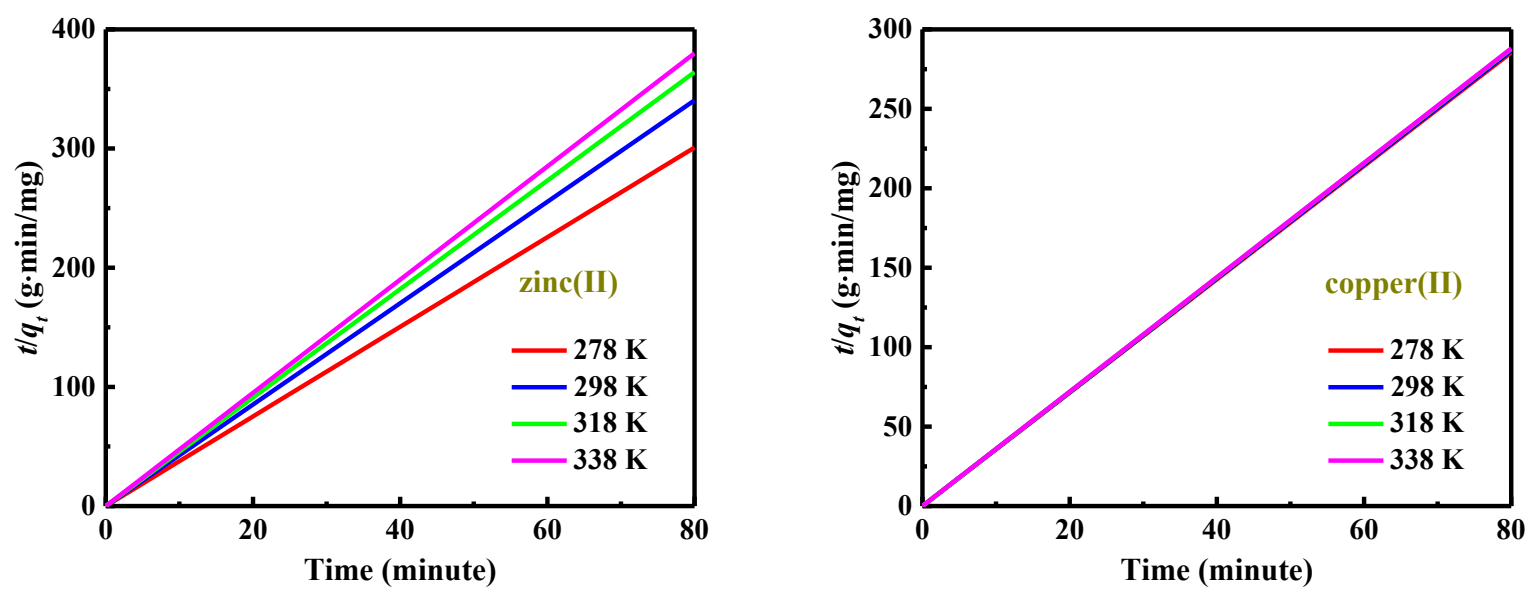

Fig. 5. Pseudo-second order plots for zinc(II) and copper(II) at different temperatures.
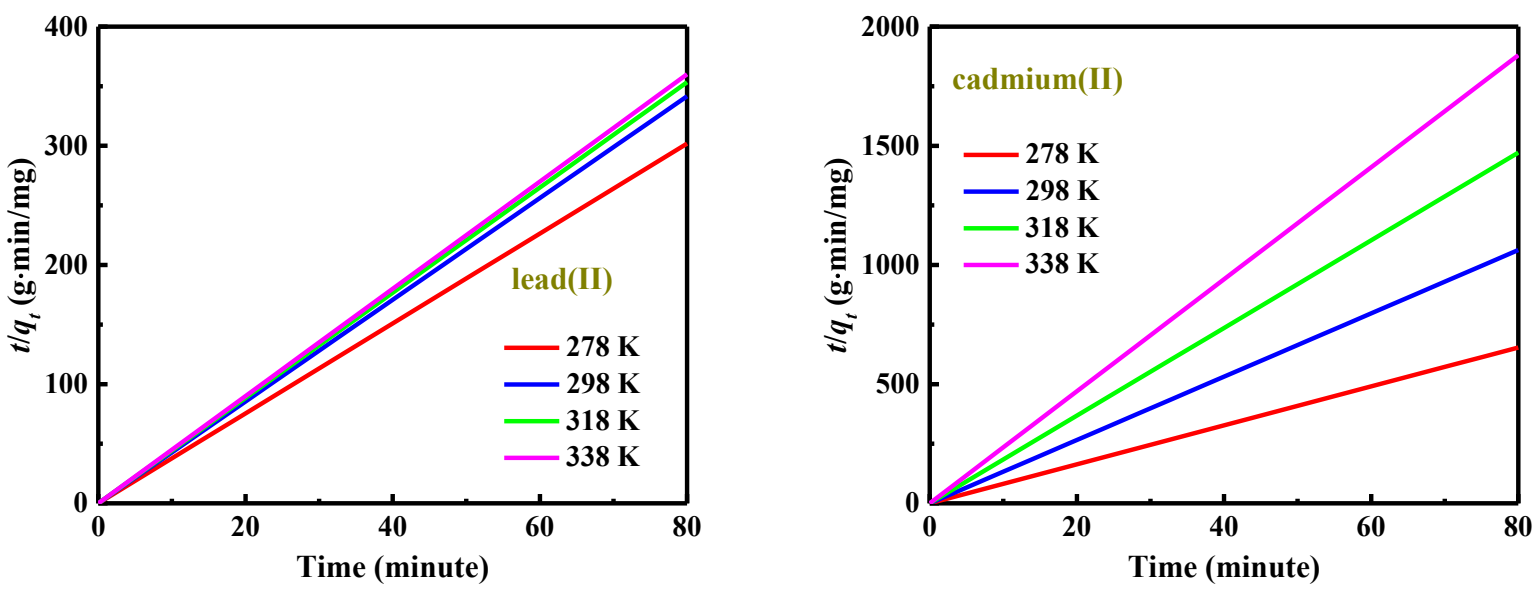

Fig. 6. Pseudo-second order plots for lead(II) and cadmium(II) at different temperatures. 


\section{Conclusion}

Kinetic study on the removal of zinc(II), copper(II), lead(II) and cadmium(II) from the aqueous solutions at different temperatures using multi-walled carbon nanotubes was carried out. Although the multi-walled carbon nanotubes used in this work were not modified (such as the modified multi-walled carbon nanotubes with 8-hydroxyquinoline used in previous work of Kosa et al. [11]), the multi-walled carbon nanotubes can successfully remove heavy metals from aqueous solutions, and their effect is not less than the modified multi-walled carbon nanotubes. Experimental results showed that increasing solution temperature can significantly improve the removal efficiency, indicating the endothermic nature of the adsorption process. The binding of the metal ions by multi-walled carbon nanotubes was found in the following order: $\operatorname{copper(II)~}>$ lead(II) $>\operatorname{zinc}$ (II) $>$ cadmium(II). The kinetics of zinc(II), copper(II), lead(II) and cadmium(II) adsorption on multi-walled carbon nanotubes were analyzed, and calculation results revealed that the adsorption of the heavy metal ions process is a pseudo-second-order process. Finally, multi-walled carbon nanotubes demonstrated that they are a promising adsorbent for the removal of heavy metal ions from aqueous solutions.

\section{Acknowledgments}

This work was supported by the National Natural Science Foundation of China (No. 51506048).

\section{References}

[1] J.-G. Yu, X.-H. Zhao, L.-Y. Yu, F.-P. Jiao, J.-H. Jiang, and X.-Q. Chen, Removal, recovery and enrichment of metals from aqueous solutions using carbon nanotubes, J. Radioanal. Nucl. Chem. 299 (2014) 1155-1163.

[2] S. Yang, J. Hu, C. Chen, D. Shao, and X. Wang, Mutual effects of $\mathrm{Pb}$ (II) and humic acid adsorption on multiwalled carbon nanotubes/polyacrylamide composites from aqueous solutions, Environ. Sci. Technol. 45 (2011) 3621-3627.

[3] G.D. Vuković, A.D. Marinković, M. Čolić, M.Đ. Ristić, R. Aleksić, A.A. Perić-Grujić, and P.S. Uskoković, Removal of cadmium from aqueous solutions by oxidized and ethylenediamine-functionalized multi-walled carbon nanotubes, Chem. Eng. J. 157 (2010) 238-248.

[4] C. Luo, R. Wei, D. Guo, S. Zhang, and S. Yan, Adsorption behavior of $\mathrm{MnO}_{2}$ functionalized multi-walled carbon nanotubes for the removal of cadmium from aqueous solutions, Chem. Eng. J. 225 (2013) 406-415.

[5] G.D. Vuković, A.D. Marinković, S.D. Škapin, M.Đ. Ristić, R. Aleksić, A.A. Perić-Grujić, and P.S. Uskoković, Removal of lead from water by amino modified multi-walled carbon nanotubes, Chem. Eng. J. 173 (2011) 855-865.

[6] C. Zhang, J. Sui, J. Li, Y. Tang, and W. Cai, Efficient removal of heavy metal ions by thiol-functionalized superparamagnetic carbon nanotubes, Chem. Eng. J. 210 (2012) 45-52.

[7] A. Stafiej and K. Pyrzynska, Adsorption of heavy metal ions with carbon nanotubes, Sep. Purif. Technol. 58 (2007) 49-52.

[8] A. Salem and R.A. Sene, Removal of lead from solution by combination of natural zeolite-kaolin-bentonite as a new low-cost adsorbent, Chem. Eng. J. 174 (2011) 619-628.

[9] Y. Li, F. Liu, B. Xia, Q. Du, P. Zhang, D. Wang, Z. Wang, and Y. Xia, Removal of copper from aqueous solution by carbon nanotube/calcium alginate composites, J. Hazard. Mater. 177 (2010) $876-880$. 
[10] M.A. Tofighy, T. Mohammadi, Adsorption of divalent heavy metal ions from water using carbon nanotube sheets, J. Hazard. Mater. 185 (2011) 140-147.

[11] S.A. Kosa, G. Al-Zhrani, and M.A. Salam, Removal of heavy metals from aqueous solutions by multi-walled carbon nanotubes modified with 8-hydroxyquinoline, Chem. Eng. J. 181-182 (2012) 159-168.

[12] Y.S. Ho and G. McKay, The kinetics of sorption of divalent metal ions onto sphagnum moss peat, Water Res. 34 (2000) 735-742.

[13] Y.-S. Ho, Second-order kinetic model for the sorption of cadmium onto tree fern: A comparison of linear and non-linear methods, Water Res. 40 (2006) 119-125.

[14]C.L. Chen, X.K. Wang, and M. Nagatsu, Europium adsorption on multiwall carbon nanotube/iron oxide magnetic composite in the presence of polyacrylic acid, Environ. Sci. Technol. 43 (2009) 2362-2367.

[15] Y.-S. Ho, Review of second-order models for adsorption systems, J. Hazard. Mater. 136 (2006) 681-689.

[16] Y.-S. Ho, Effect of $\mathrm{pH}$ on lead removal from water using tree fern as the sorbent, Bioresour. Technol. 96 (2005) 1292-1296.

[17] K.V. Kumar, Pseudo-second order models for the adsorption of safranin onto activated carbon: Comparison of linear and non-linear regression methods, J. Hazard. Mater. 142 (2007) 564-567. 